\title{
Assessment Quality of Life in Patient with Lip Cancer after Radical Surgery and Radiation Therapy
}

\author{
Slipetsky Roman ${ }^{1}$, Rajesh Pandit ${ }^{1}$ and Halay Oleh $^{2}$ \\ 1. Danylo Halytsky Lviv National Medical University, Pekarska Street 69, Lviv 79010, Ukraine \\ 2. Lviv Oncological Regional Treatment and Diagnostic Centre, Department of Head and Neck Tumors, Hascheka Str. 2b, Lviv city \\ 79010, Ukraine
}

\begin{abstract}
Lip cancer is one of the most frequent malignant neoplasms of the oral cavity. It has wide impact on the quality of life for patient and their families. In oncology, assessing quality of life is being used as a primary outcome measure to study the effectiveness of treatment therapy for cancer patient. Material and methods: This study included total of 103 patients affected by lip cancer (stage I-IV) who underwent treatment (surgery and radiation therapy) from 2010-2016 at Lviv Regional Oncological Centre, Department of Head and Neck Oncology, Lviv city. Quality of life (QOL) was assessed by using EORTC QLQ C30 V.30 Questionnaires and Head and Neck Cancer Specific Modules EORTC QLQ H\&N35. All patients were divided into two groups, in which group-I consisted of 30 patients after radical surgery and in group-II consisted of 73 patients after radical radiation therapy. The QOL was assessed during one month after treatment of lip cancer. Result: In group-I (radical surgery therapy) 43\% of patients had locally advanced disease with local metastasis to neck lymph nodes in 13\% of cases. In this group G3 histological differentiation was strongly correlated with advance disease and poor prognosis. In group-II (radical radiation therapy) 88\% of patients had mostly local disease (stages I-II). EORTC QLQ C30 and H\&N35 score were better in group-II (radical radiation therapy) before treatment, than in group-I (radical radiation therapy). But one month after treatment group-I has better European Organization for Research and Treatment of Cancer (EORTC) score. Kaplan-Meier curve also shows better survival rate in group-I patients. Conclusion: Our results suggest that patient's quality of life is better when treated with radical surgery therapy and it also improves survival rate as compared with radical radiation therapy.
\end{abstract}

Key words: QOL, surgical therapy, radiation therapy, lip cancer, EORTC.

\section{Introduction}

Lip cancer is a type of oral cancer that develops at the junction of the oral cavity and the skin; it is most common on the lower lip (80\%), while the upper lip and commissures are involved less often (5-8\% and $7-15 \%$ of cases, respectively) [1, 2]. Lip cancer is commonly developed on the vermilion border of the lip and is diagnosed as squamous cell carcinoma (SCC) in $95 \%$ of cases, basal cell carcinomas and adeno carcinomas occur more rarely [1]. Squamous cell carcinoma (SCC), the most prevalent type of lesion in head and neck, is particularly common in the oral cavity [3, 4]. Malignantoral tumors are generally

Correspondence author: Rajesh Pandit, Dr., research field: general medicine. associated with poor prognosis and a 5-year survival rate of up to $50 \%$, especially due to late diagnosis [5], although, the prognosis is dependent mainly on the clinical staging of the tumor, especially with regard to its size and lymphnode status [6]. The etiology of lower lip SCC is highly related to chronic sunlight (especially UVB) exposure and other factors, such as low sociodemographic conditions, genetic susceptibility, and immune suppression, which might produce a synergistic effect [2, 6, 7]. However, it is very important to note that lip cancer may evolve from pre-cancerous lesion of the lip, such as actinic cheilitis or from healthy lip [7].

The World Health Organization (WHO) considers quality of life equal to the individual's perception of their position in life in the context of the culture and 
value systems in which they live and in relation of their goals, expectations, standards, and concerns. This definition also includes the aspects of physical well-being, personal well-being, social and functional activities, and economic influences [8]. Patient-related quality of life (QOL) is increasingly used by clinicians to assist determining success following the treatment of oral cancer $[9,10]$. Functional, aesthetic, emotional and social factors - all play apart in determining QOL, and should therefore receive appropriate attention in treatment planning and rehabilitation [11]. Several QOL questionnaires have been proposed, including the EORTC (European Organization for Research and Treatment of Cancer), the FACT (Functional Assessment of Cancer Therapy) and the UW-QOL (University Washington Quality of Life questionnaire), which takes general well-being into account $[9,12]$. The European Organization for Research and Treatment of Cancer (EORTC) questionnaires have been used in numerous studies to evaluate the QOL of cancer patients. This usually involves combining a general questionnaire, QLQ-Core-30 (version 3.0), which evaluates those areas common to different tumours and treatments, complemented by different modules which assess the aspect specific to a particular cancer and there treatments. One of these modules is QLQ- H\&N35 designed for patients with head and neck cancers. The combination of these surveys gives the ability to identify disease-specific side effects [11]. The most important outcome for cancer patients is overall survival, which is also a primary end point in quality of life. Factors that exert a major influence on cancer survival are a delay in diagnosis and less effective treatment at advanced stage of cancer [13]. However, the overall survival of cancer patients has improved over the years as a result of advancements in prevention, diagnosis and treatment [14].

\section{Epidemiology}

Oral cancer is a serious and growing problem in many parts of the world. Oral and pharyngeal cancer, grouped together, is the sixth most common cancer worldwide. According to the European Cancer Observatory (ECO), the estimated number of new cases of lip, oral and pharyngeal cancer in Europe was 99,630 in 2012, and the estimated number of deaths from these cancers was 43,662 [15]. Overall in the EU oral and pharyngeal cancer occupies the seventh position [16]. The lifetime risk of developing oral and pharyngeal cancer in Europeans is estimated at 1.85\% for men and $0.37 \%$ for women. The incidence rates are higher in Eastern Europe compared with Western, Northern or Southern Europe. Within the EU countries the highest male incidence rates are found in France and Hungary [17]. A comparison of incidence and mortality rate among Europeans and Ukraine is shown in Fig. 1.

\section{Materials and Methods}

The purpose of this cross-sectional, prospective study is to obtain more specific information about quality of life in patients with lip cancer by EORTCQOL-C30 and QOL-H\&N35 as a predictor of effectiveness of treatment and survival rate. Patients received questionnaires (in Ukrainian version) during check-up at our OPD, Lviv Oncological Regional Treatment and Diagnostic Centre, Department of Head and Neck Tumors, and then patients were asked to fill out the questionnaires pre-operatively (after the histopathological diagnosis and one month after treatment). The questionnaires were individual and self-administered, although, instructions regarding filling-out the questionnaires and the scales were explained to the participants, by resident doctor and they were asked to answer each question according to the point that there was no external influence on the patient, while filling the questionnaires. This study included a total of 103 patients affected by squamous cell carcinoma of the lip (lower/upper lip) at stage I-IV, who underwent radical surgery and radical radiation therapy, between 2010-2016 at Lviv 

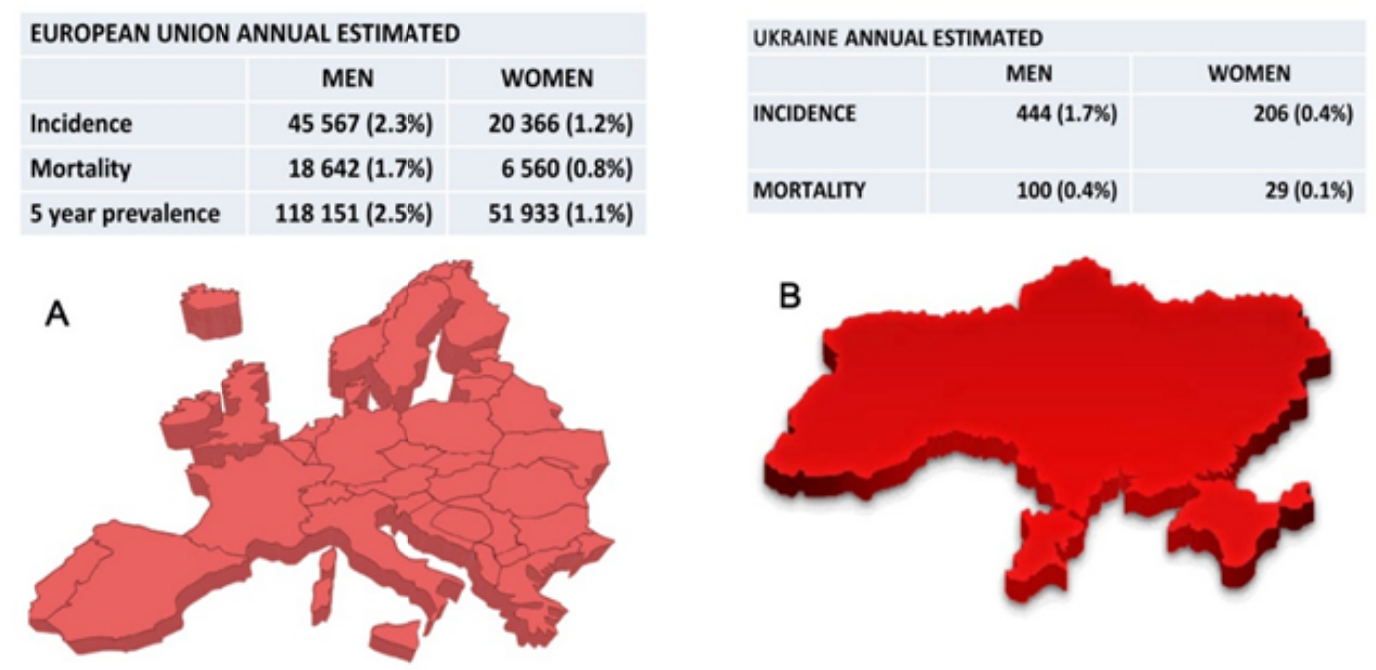

Fig. 1 (a) Globocan 2012 (IARC) section of cancer surveillance; (b) Bulletin of National Cancer Registry of Ukraine No. 18, 2015-2016.

Oncological Regional Treatment and Diagnostic Centre, Department of Head and Neck Tumors, Lviv city, Ukraine. All patients were staged and treated under the guidelines of NCCN Clinical practice guideline in oncology [18]. Here treatment also depended on patient's choice after explaining about the options of treatment (surgical, radiation or both) and compliance of patients with medical professionals.

The analysis of the questionnaires was done in accordance with EORTC guidelines. All patients were divided into two groups in which group-I consisted of 30 patients after radical surgery and group-II consisted of 73 patients after radiation therapy. The QOL was assessed during one month after treatment of cancer.

The data were obtained from the oncology clinic of lip cancer. All the data were stored in Microsoft Office Excel 2016, and statistical analysis was made using the software STATISTICAV.10 and IBMSPSSV.22. Comparison soft two groups were performed with non-parametric Manne-Whitney U-test. Significance was established as $p<0.05$ and survival analysis was performed by a Kaplan-Meier regression curve. The data were presented in three blocks:

(1) EORTC QLQ-C30/functional scale;
(2) EORTC QLQ-C30/symptoms scales and single items;

(3) EORTC QLQ-H\&N C35 module.

Relation between parameters was exam in edusing Pearson's chi-square test $(p<0.05)$ for dichotomized variables.

\section{Results}

A total of 103 patients, sociodemographic factors such as age, gender, marital status, comorbidity and clinical factors such as tumour site and stage, metastasis and quality of life were collected and analysed. Average patients age in group-I was $71.0 \pm$ 2.3 years with sex distribution of $77 \%$ male and $23 \%$ female. And in group-II, was $69.8 \pm 1.4$ years, with sex distribution of $74 \%$ men and $26 \%$ women. In group-I (radical surgery therapy) 43\% (T1-1, T2-16, T3-9, T4-4) of patients had locally advanced disease with local metastasis to neck lymph nodes in $35 \%(\mathrm{~N} 0-26, \mathrm{~N}+-4)$ of cases. With regard to TNM staging, there were 15 patients with stage II (50\%), 11 patients with stage III (37\%), and 4 patients with stage IV (13\%). In histological classification, 12 patients had low grade (G1), 14 patients had intermediate-grade (G2) and 4 had high-grade (G3) SCC. In groups with G3, histological differentiation 
Table 1 (A) Comparison of EORTC QLQ-C30 quality of life in relation to treatment received (Manne-Whitney U Test); (B) Comparison of EORTC QLQ-H\&N35 quality of life in relation to treatment received (Manne-Whitney $U$ Test).

\begin{tabular}{|c|c|c|c|c|c|c|c|}
\hline \multirow{2}{*}{ A. EORTC QLQ-C30 } & \multicolumn{3}{|c|}{ Surgery $(n=30)$} & \multicolumn{3}{|c|}{ Radiology $(n=73)$} & \\
\hline & Mean \pm SD & Median & Range & Mean \pm SD & Median & Range & \\
\hline Functional scales & $69.01 \pm 20.96$ & 66.66 & $13.33-100$ & $87.89 \pm 14.31$ & 91.66 & $16.66-100$ & $<0.001$ \\
\hline Physical functioning & $69.77 \pm 24.93$ & 73.33 & $13.33-100$ & $85.66 \pm 13.68$ & 86.66 & $46.66-100$ & 0.002 \\
\hline Role functioning & $63.89 \pm 20.10$ & 66.66 & $33.33-100$ & $93.84 \pm 10.94$ & 100 & $66.66-100$ & $<0.001$ \\
\hline Emotional functioning & $67.50 \pm 19.74$ & 70.83 & $25.00-100$ & $90.07 \pm 8.75$ & 91.66 & $66.66-100$ & $<0.001$ \\
\hline Cognitive functioning & $68.33 \pm 14.08$ & 66.66 & $33.33-100$ & $87.90 \pm 17.84$ & 100 & $16.66-100$ & $<0.001$ \\
\hline Social functioning & $75.56 \pm 23.87$ & 66.66 & $33.33-100$ & $81.96 \pm 15.90$ & 83.33 & $33.33-100$ & 0.294 \\
\hline Global health scale & $41.11 \pm 18.69$ & 41.66 & $0-75$ & $31.39 \pm 21.18$ & 25 & $8.33-83.33$ & 0.015 \\
\hline Symptom scales & $28.02 \pm 23.98$ & 22.22 & $0-100$ & $12.53 \pm 13.59$ & 11.11 & $0-66.66$ & $<0.001$ \\
\hline Fatigue & $39.63 \pm 24.18$ & 33.33 & $0-100$ & $12.02 \pm 12.53$ & 11.11 & $0-66.66$ & $<0.001$ \\
\hline Nausea and Vomiting & $11.11 \pm 15.37$ & 0 & $0-66.66$ & $13.01 \pm 13.68$ & 16.66 & $0-50$ & 0.402 \\
\hline Pain & $33.33 \pm 21.88$ & 33.33 & $0-83.33$ & $12.56 \pm 14.64$ & 16.66 & $0-50$ & $<0.001$ \\
\hline \multicolumn{8}{|l|}{ Single-item questions } \\
\hline Dysponoea & $27.77 \pm 27.80$ & 33.33 & $0-100$ & $5.48 \pm 12.44$ & 0 & $0-33.33$ & $<0.001$ \\
\hline Insomnia & $35.55 \pm 26.16$ & 33.33 & $0-100$ & $10.96 \pm 18.47$ & 0 & $0-66.66$ & $<0.001$ \\
\hline Loss of appetite & $34.44 \pm 26.96$ & 33.33 & $0-100$ & $11.42 \pm 16.87$ & 0 & $0-66.66$ & $<0.001$ \\
\hline Constipation & $5.55 \pm 17.69$ & 0 & $0-66.66$ & $5.94 \pm 12.84$ & 0 & $0-33.33$ & 0.601 \\
\hline Diarrhoea & $4.44 \pm 11.52$ & 0 & $0-33.33$ & $2.28 \pm 8.48$ & 0 & $0-33.33$ & 0.609 \\
\hline Financial difficulties & $27.77 \pm 31.66$ & 16.66 & $0-100$ & $22.83 \pm 24.14$ & 33.33 & $0-100$ & 0.671 \\
\hline \multirow{2}{*}{ B. EORTC QLQ- H\&N35 } & \multicolumn{3}{|c|}{ Surgery $(n=30)$} & \multicolumn{3}{|c|}{ Radiology $(n=73)$} & \\
\hline & Mean \pm SD & Median & Range & Mean \pm SD & Median & Range & \\
\hline Scales & $21.90 \pm 19.74$ & 16.66 & $0-100$ & $24.52 \pm 15.41$ & 25 & $0-66.66$ & 0.058 \\
\hline Pain & $35.00 \pm 17.01$ & 33.33 & $8.33-75.0$ & $29.22 \pm 12.89$ & 25 & $0-58.33$ & 0.178 \\
\hline Swallowing & $23.61 \pm 17.92$ & 25 & $0-66.66$ & $31.39 \pm 10.62$ & 33.33 & $8.33-66.66$ & 0.002 \\
\hline Senses problems & $17.22 \pm 20.29$ & 16.66 & $0-66.66$ & $29.22 \pm 13.83$ & 33.33 & $0-66.66$ & $<0.001$ \\
\hline Speech problems & $13.33 \pm 12.85$ & 11.11 & $0-44.44$ & $20.85 \pm 10.30$ & 22.22 & $0-44.44$ & 0.002 \\
\hline Trouble with social eating & $35.28 \pm 20.14$ & 33.33 & $16.66-100$ & $37.56 \pm 12.96$ & 33.33 & $0-66.66$ & 0.067 \\
\hline Trouble with social contact & $23.33 \pm 17.13$ & 20 & $0-66.66$ & $22.47 \pm 7.74$ & 20 & $6.66-40$ & 0.916 \\
\hline Less sexuality & $5.55 \pm 13.37$ & 0 & $0-50$ & $0.91 \pm 5.48$ & 0 & $0-33.33$ & 0.270 \\
\hline \multicolumn{8}{|l|}{ Single-item questions } \\
\hline Teeth & $45.55 \pm 30.93$ & 33.33 & $0-100$ & $38.36 \pm 24.64$ & 33.33 & $0-100$ & 0.256 \\
\hline Maximum mouth opening & $45.55 \pm 33.31$ & 33.33 & $0-100$ & $33.33 \pm 21.52$ & 33.33 & $0-66.66$ & 0.091 \\
\hline Dry mouth & $33.33 \pm 24.76$ & 33.33 & $0-100$ & $56.16 \pm 20.70$ & 66.66 & $33.33-100$ & $<0.001$ \\
\hline Sticky saliva & $33.33 \pm 26.26$ & 33.33 & $0-100$ & $61.19 \pm 22.92$ & 66.66 & $33.33-100$ & $<0.001$ \\
\hline Cough & $20.00 \pm 18.77$ & 33.33 & $0-66.66$ & $28.31 \pm 17.28$ & 33.33 & $0-66.66$ & 0.072 \\
\hline Felt ill & $43.33 \pm 36.25$ & 33.33 & $0-100$ & $44.75 \pm 28.98$ & 33.33 & $0-100$ & 0.805 \\
\hline Use of pain killers & $56.66 \pm 50.40$ & 100 & $0-100$ & $31.51 \pm 46.78$ & 0 & $0-100$ & 0.046 \\
\hline Use of nutritional supplements & $43.33 \pm 50.40$ & 0 & $0-100$ & $4.11 \pm 20.00$ & 0 & $0-100$ & 0.002 \\
\hline Use of feeding tube & $20.0 \pm 40.68$ & 0 & $0-100$ & 0 & 0 & 0 & 0.113 \\
\hline Weight loss & $43.33 \pm 50.40$ & 0 & $0-100$ & $41.10 \pm 49.54$ & 0 & $0-100$ & 0.862 \\
\hline Weight gain & $6.66 \pm 25.37$ & 0 & $0-100$ & $36.99 \pm 48.61$ & 0 & $0-100$ & 0.016 \\
\hline
\end{tabular}

was strongly correlated with advanced disease $144(r$ $=0.89, p<0.05)$ and poor prognosis $(r=0.72, p<$ $0.05)$. Regarding the tumour localization, 1 patient with upper-lip (3.3\%) and 29 patients with lower lip
$(96.9 \%)$ in which selective lymph dissection of the I-III level from both sides was performed in 10 cases $(33 \%)$, resection of the lip following plastic surgery (different skin flaps) - 26 cases $(87 \%)$ and extended 
dissection (lip + cheek + mandibular resection) - in 3 cases (10\%). In group-II (radical radiation therapy) $88 \%$ of patients had mostly local disease (stages I-44, stage II-20, stage III-19) with regard to TNM staging. The results of the questionnaire are listed in Table 1.

\subsection{EORTC QLQ-C30/Functional Scales (FS)}

The functional scales $(p<0.001)$ were 69.01 (Mean) \pm 20.96 (SD) in surgery group and 87.89 (Mean) \pm 14.31 (SD) in radiation with 100 standing for uncompromised functioning. Physical functional scales ( $p=0.002)$ with different mean express a good ability to progress daily activities in surgical group $(69.77 \pm 24.93)$ which is highly significant than the radiation group $(85.66 \pm 13.68)$. But the role functioning ( $p<0.001$ ), emotional functioning ( $p<$ $0.001)$ and cognitive functioning $(p<0.001)$ increased constantly in surgical group, whereas in radiation group, these symptoms progressively decreased with a high significant difference. The social function showed no significant changes in both groups.

The estimation of global health status $(p=0.015)$ parameter for overall QOL was statistically significant. In surgical group, the average values were $41.11 \pm$ 18.69 with the range of 0-75 and in radiation group, it was $31.39 \pm 21.18$ with the range of 8.3-83.33, which increased significantly towards end of the study.

\subsection{EORTC QLQC-30/Symptoms Scales (SS) and Single Items}

The symptoms scale $(p<0.001)$ was 28.02 (Mean) \pm 23.98 (SD) in surgery group and 12.53 (Mean) \pm 13.59 (SD) in radiation group both on a scale of 100 , in which 0 indicates "no" and "100" indicates maximum symptoms. Fatigue $(p<0.001)$ and pain $(p$ $<0.001$ ) showed a significant improvement with low score in surgical group, while problems with nausea and vomiting ( $p=0.402$ ) were equally at a low score in both groups. Single items scale symptoms like dyspnea $(p<0.001)$, insomnia $(p<0.001)$ and loss of appetite $(p<0.001)$ also showed worse score in surgical group than in radiation group, with high significant difference, but symptoms were seen improving at the end of study mostly in surgical group. The financial difficulties $(p=0.067)$, diarrhea $(p=$ $0.609)$ and constipation ( $p=0.601)$ had no changes in both groups without any significant differences.

\subsection{EORTC QLQ-H\&N C35}

The symptoms scale in head and neck area was 21.90 (Mean) \pm 19.74 (SD) in surgery group and 24.52 (Mean) \pm 15.41 (SD) in radiation group, but score scale varied in different symptoms for both groups as shown in Table 1 . In which sense problem ( $p<0.001)$, swelling and speech $(p=0.002)$ had a significant difference in radiation group. Pain ( $p=$ 0.178 ), trouble with social eating ( $p=0.067)$, trouble with social contact $(p=0.916)$ and less sexuality ( $p=$ 0.270 ) in head and neck area remained the same in both groups with no significant changes.

Single items scale with symptoms like dry mouth $(p$ $<0.001)$ and sticky saliva $(p<0.001)$ gradually improved in surgical group than in radiation group (due to irritation of mucosa by radiation in the surrounding area) with a high significant difference. Teeth $(p=0.256)$, maximum mouth opening (0.091), cough $(p=0.072)$, felt ill ( $p=0.805)$, use of feeding tube ( $p=0.046)$ and weight loss $(p=0.862)$ remain unchanged in both groups, but maximum mouth opening is better in surgical group with $45.55 \pm 33$.31. Use of pain killer $(p=0.046)$ has low score in radiation group (31.51 \pm 33.31$)$ than in surgical group (56.66 \pm 50.40$)$ with significant changes. Lastly, use of nutritional supplements ( $p=0.002$ ) score is very high in surgical group (43.33 \pm 50.40$)$, when compared with radiation group $(4.11 \pm 20.0)$ which is significantly increased with balance nutrition and improved patient's health related condition drastically after surgery.

Overall evaluation of EORTCQLQC 30 and H\&N35 scale, score was better in group-II (radical radiation therapy) before treatment ( $p<0.05$ ), than in 


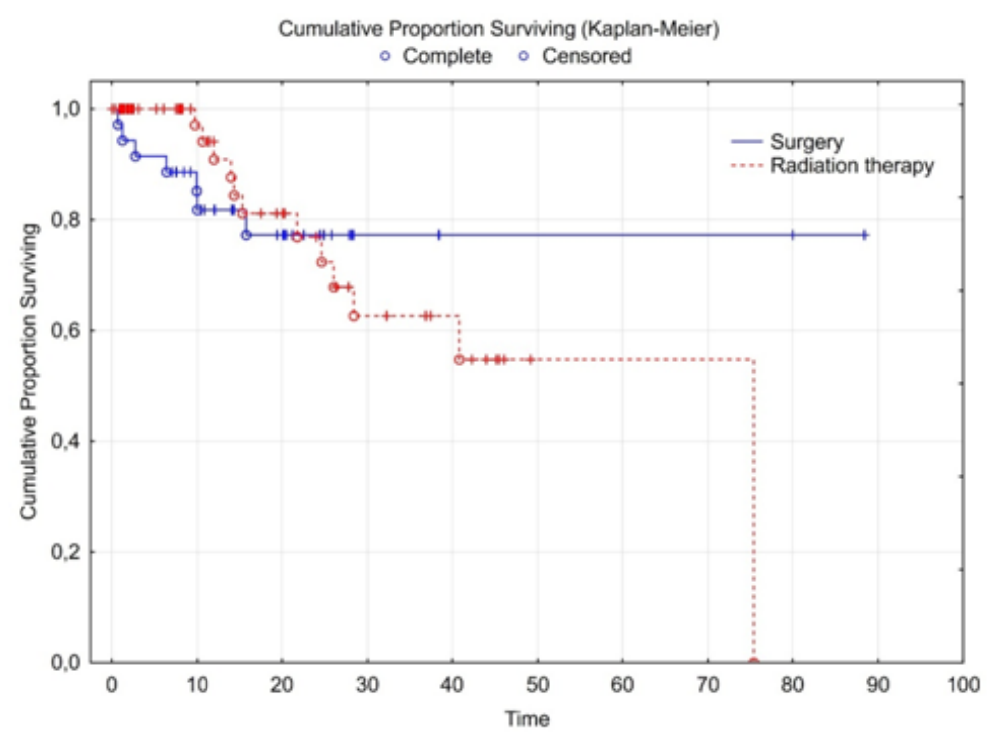

Fig. 2 Kaplan Meier curve also show better survival rate in group-I (radical surgery therapy) (p<0.05) patients.

group-I (radical surgery therapy). But one month after treatment group-I (radical surgery therapy) $(p<0.05)$ had better EORTC score. Kaplan-Meier curve also showed better survival rate in group-I (radical surgery therapy) ( $p<0.05)$ patients, shown in Fig. 2.

\section{Discussion}

There are different questionnaires available for assessing the QOL in this study; we used the EORTC 30 as a generic questionnaire and $\mathrm{H} \& \mathrm{~N} 35$ as a disease specific questionnaire. Comparison of treatment between radiation and surgical method is very important in this study, because it shows that our patients' QOL has an excellent survival rate, after undergoing surgery therapy depending upon the stages of lip tumor.

The result of the present evaluation with the EORTC instrument showed that surgical therapy of lip cancer had constantly improved role functioning, emotional functioning and cognitive functioning, while social functioning appeared to be affected during the observation period in both groups. It can also affect physical and psychological behaviour that can lead to social isolation and decrease the quality of life. The higher scores of pretreatment physical function and augmentation in the overall QOL in the 6 months after treatment were associated with increased survival in patients with $\mathrm{H} \& \mathrm{~N}$ cancer [12].

In our study, pain and fatigue scores improved significantly after surgery, pain was also considered important for QOL especially in the pre-treatment evaluation. The patients with the lowest overall QOL scores tend to attribute the greatest importance to pain [19]. Also in pre-treatment evaluation, the priorities identified by the patient's survival were the probability of cure, longevity and the absence of pain [12, 19].

In this study, only 30 patients with lip cancer agreed to undergo surgical therapy; because surgical treatment usually includes resection and results in loss of functioning tissue, resulting in importance of appearance and speech function.

On the other hand, surgery has beneficial effect like it allows a histological control of the excision specimen and allows asserting. The surgical margin varies according to histological type [20]. Surgery is the treatment of choice, despite the fact that, for less extensive lesions in the early stages (T1/T2N0), the results obtained with radiotherapy (RT) may compare favourably [21, 22].

Whereas in radiation therapy, 1 month after treatment, patients showed more physical deterioration, increase in senses problem, speech problem trouble 
with social contact and eating according to the questionnaires, which can be explained by the adverse effects of radiation therapy. Head and neck RT may offer better functional and cosmetic result, but can lead to complications, with significant impairment of patient QOL [23, 24]. Several strategies have been used to prevent oral complication because the treatment of these conditions is still considered difficult [23, 25]. Almost all the adverse effects can be improved by dental rehabilitation in both groups, but it was difficult to avail dental rehabilitation to all patients in our study due to social and financial reason.

Overall, the main differences between the groups were that the survivors after surgical therapy have high scores in EORTC C-30 (example: functional scales and global health status) and EORTC H\&N 35 (example: dry mouth, sticky saliva and use of nutrition). It is also reasonable for comparing between surgical and radiation therapy because patients were from the same geographical region who had undergone treatment in the same oncological clinic and there were marked similarities in gender and age.

The result obtained from EORTC questionnaires, showed the consequence of treatment in both the groups (surgical and radiation) had an important influence on QOL. It also showed that QOL in lip cancer is difficult to evaluate in respect of size and tumor stage, resection, reconstruction and after radiation therapy, because they are inter-related and interdependent on each other. There are other factors that can be also an influence to the QOL, which were not taken for evaluation of statistical data in this study. Different risk factors have been associated with lip tumors such as age, chronic exposure to solar radiation and tobacco and alcohol habits. It is very important to note that lip cancer may evolve from pre-cancerous lesions of the lip, such as actiniccheilitis, or from healthy lips [7]. These risk factors are difficult to evaluate by using this questionnaire.

\section{Conclusions}

Health-related quality-of-life issues are paramount in head and neck cancers. The treatment of lip cancer is governed by the stage of disease and the choice of local treatment is based on the expected functional and cosmetic outcome. Our results suggest that patient's quality of life is better when treated with radical surgery therapy and it also improved survival rate as compared to radical radiation therapy.

\section{Acknowledgement}

We would like to thank residents and staff at Lviv Oncological Regional Treatment and Diagnostic Centre, Department of Head And Neck Tumors for their support and for allowing us an access to their clinical service. An initial version of this article has been presented as conference abstract in 13th Warsaw International Medical Congress Abstract Book according to the following link: http://wimc.wum.edu.pl/wpcontent/uploads/2017/05/1 3th-Warsaw-International-Medical-Congress-Abstract -Book.pdf. This article is also presented as conference abstract in 28th European Students Conference Abstract Book according to the following link: https://drive.google.com/file/d/1RtzlEcQt9YDbw7lC GfYTPkCZ8H1JSqM/view?usp=sharing.

\section{Funding}

None to declare.

\section{Conflicts of Interest}

The authors have no conflict of interest to report.

\section{Ethical Approval}

All procedures performed in studies involving human participants were in accordance with the ethical standards of the institutional and/or national research committee.

\section{Data Availability}

The data sets used to support the finding of this 
study are not publicly available because this original research article is not published in any journal yet, but are available from the corresponding author on reasonable request.

\section{References}

[1] Czerninski, R., Zini, A., and Sgan Cohen, H. D. 2010. "Lip Cancer: Incidence, Trends Histology and Survival: 1970-2006.” Br J Dematol 162: 1103-9.

[2] Biasoli, E. R., Valente, V. B., Mantovan, B., et al. 2016. "Lip Cancer: A Clinic Opathological Study and Treatment Outcomes in a 25-Year Experience." J Oral Maxillofac Surg 74 (7): 1360-7.

[3] Rao, S. V. K., Mejia, G., Roberts-Thomson, K., and Logan, R. 2013. "Epidemiology of Oral Cancer in Asia in the Past Decade-An Update (2000-2012)." Asian Pac J Cancer Prev 14: 5567-77.

[4] Rana, M., Kanatas, A., Herzberg, P. Y., et al. 2015. "Prospective Study of the Influence of Psychological and Medical Factors on Quality of Life and Severity of Symptoms among Patients with Oral Squamous Cell Carcinoma.” Br J Oral Maxillofac Surg 53: 364-70.

[5] Lambert, R., Sauvaget, C., De Camargo Cancela, M., and Sankaranarayanan, R. 2011. "Epidemiology of Cancer from the Oral Cavity and Oropharynx." Eur $J$ Gastroenterol Hepatol, 23633-41.

[6] Ozkul, Y., Songu, M., Imre, A., et al. 2016. "Early Stage Squamous Cell Carcinoma of the Lower Lip: Predictive Factors for Recurrence." J Laryngol Otol 130 (4): $369-72$.

[7] Viera, R. A., Minicucci, E. M., Marques, M. E., and Marques, S. A. 2012. "Actinic Cheilitis and Squamous Cell Carcinoma of the Lip: Clinical, Histopathological and Immunogenetic Aspects." An Bras Dermatol 87 (1): 105-14.

[8] Kumar, P., Alvi, H. A., Rao, J., Singh, B. P., Jurel, S. K., Kumar, L., et al. 2013. "Assessment of the Quality of Life in Maxillectomy Patients: A Longitudinal Study." $J$ Aadv Prosthodont 5: 29-35.

[9] Rathod, S., Livergant, J., Klein, J., Witterick, I., and Ringash, J. 2015. "A Systematic Review of Quality of Life in Head and Neck Cancer Treat with Surgery with or without Adjuvant Treatment." Oral Oncology 51: 888-900.

[10] Klug, C., Neuburg, J., Glaser, C., Schwarz, B., Kermer, C., and Millesi, W. 2002. "Quality of Life 2-10 Years after Combined Treatment for Advanced Oral and Oropharyngeal Cancer." International Journal of Oral and Maxillofacial Surgery 31: 664-9.

[11] López-Jornet, P., Camacho-Alonso, F., López-Tortosa, J., Palazon Tovar, T., and Rodríguez-Gonzales, M. A. 2012.
"Assessing Quality of Life in Patients with Head and Neck Cancer in Spain by Means of EORTC QLQ-C30 and QLQ-H\&N35." Journal of Cranio-Maxillo-Facial Surgery 40: 614-20.

[12] Rogers, S. N., Hogg, E. S., Cheung, W. K., et al. 2015. "The Use of Health Related Quality of Life Data to Produce Information Sheets for Patients with Head and Neck Cancer." Ann R Coll Surg Engl 97: 359-63.

[13] Brocklehurst, P., Kujan, O., O’Malley, L. A., Ogden, G., Shepherd, S., and Glenny, A. 2013. "Screening Programmes for the Early Detection and Prevention of Oral Cancer." Cochrane Database Syst Rev 11: CD004150.

[14] Bower, J. E., Bak, K., Berger, A., Breitbart, W., Escalante, C. P., Ganz, P. A., Schnipper, H. H., Lac-Chetti, C., Ligibel, J. A., and Lyman, G. H. 2014. "Screening, Assessment, and Management of Fatigue in Adult Survivors of Cancer: An American Society of Clinical Oncology Clinical Practice Guideline Adaptation." Journal of Clinical Oncology 32: 1840-50.

[15] Ferlay, J., Steliarova-Foucher, E., Lortet-Tieulent, J., Rosso, S., Coebergh, J. W. W., Comber, H., Forman, D., and Bray, F. 2013. "Cancer Incidence and Mortality Patterns in Europe: Estimates for 40 Countries in 2012." European Journal of Cancer 49:1374-403.

[16] Boyle, P., and Ferlay, J. 2005. "Cancer Incidence and Mortality in Europe, 2004." Annals Oncology 16: 481-8.

[17] Warnakulasuriya, S. 2010. "Living with Oral Cancer: Epidemiology with Particular Reference to Prevalence and Life-Style Changes that Influence Survival." Oral Oncology 46: 407-10.

[18] 2018. NCCN Clinical Practice Guidelines in Oncology (NCCN Guidelines): Head and Neck Cancers, ver. 2, June 20, 2018.

[19] Rogers, S. N., Laher, S. H., Overend, L., and Lowe, D. 2002. "Importance-Rating Using the University of Washington Quality of Life Questionnaire in Patients Treated by Primary Surgery for Oral and Oro-Pharyngeal Cancer." J Craniomaxillofac Surg 30: 125-32.

[20] Staub, G., Revol, M., May, P., Bayol, J. C., Verola, O., et al. 2008. "Excision Skin Margin and Recurrence Rate of Skin Cancer: A Prospective Study of 844 Cases." Annalesde Chirurgie Plastiqueet. Esthetique. 53: 389-98.

[21] Guibert, M., David, I., Vergez, S., et al. 2011. "Brachy Therapy in Lip Carcinoma: Long-Term Results." Int $J$ Radiat Oncol Biol Phys 81 (5): e839-43.

[22] Thanh Pham, T., Cross, S., Gebski, V., and Veness, M. J. 2015. "Squamous Cell Carcinoma of the Lip in Australian Patients: Definitive Radiotherapy Is an Efficacious Option to Surgery in Select Patients." Dermatol Surg 41 (2): 219-25.

[23] Migliorati, C. A., Seneda, L. M., and Burton, E. L. 2015. 
"Oral Complications of Cancer Therapy: A Summary Guide for the Clinician." J Tenn Dent Assoc 95 (1): 24-32.

[24] Verrone, J. R., Alves, F. A., Prado, J. D., et al. 2013. "Impact of Intraoral Stent on the Side Effects of Radiotherapy for Oral Cancer." Head Neck 35 (7):
E213-7.

[25] Verrone, J. R., Alves, F. A., Prado, J. D., et al. 2014. "Benefits of an Intraoral Stent in Decreasing the Irradiation Dose to Oral Healthy Tissue: Dosimetric and Clinical Features." Oral Surg Oral Med Oral Pathol Oral Radiol 118 (5): 573-8. 